\title{
Oblivious Evaluation of Non-deterministic Finite Automata with Application to Privacy-Preserving Virus Genome Detection
}

\author{
Hirohito Sasakawa \\ Hokkaido University \\ sasakawa@ist.hokudai.ac.jp \\ Hiroki Arimura \\ Hokkaido University \\ arim@ist.hokudai.ac.jp
}

\author{
Hiroki Harada \\ University of Tsukuba \\ h.hiroki@mdl. \\ cs.tsukuba.ac.jp \\ Koji Tsuda \\ University of Tokyo/AIST/JST \\ tsuda@k.u-tokyo.ac.jp
}

\author{
David A. duVerle \\ University of Tokyo/AIST \\ dave.duverle@aist.go.jp \\ Jun Sakuma \\ University of Tsukuba/JST \\ jun@cs.tsukuba.ac.jp
}

\begin{abstract}
Various string matching problems can be solved by means of a deterministic finite automaton (DFA) or a non-deterministic finite automaton (NFA). In non-oblivious cases, DFAs are often preferred for their run-time efficiency despite larger sizes. In oblivious cases, however, the inevitable computation and communication costs associated with the automaton size are more favorable to NFAs. We propose oblivious protocols for NFA evaluation based on homomorphic encryption and demonstrate that our method can be orders of magnitude faster than DFA-based methods, making it applicable to real-life scenarios, such as privacy-preserving detection of viral infection using genomic data.
\end{abstract}

\section{Categories and Subject Descriptors}

k.6 [Management of Computing and Information Systems]: Security and Protection; F.2.2 [Nonnumerical Algorithms and Problems]: Pattern matching

\section{General Terms}

Algorithms; Experimentation; Security

\section{Keywords}

Oblivious NFA evaluation; regular expression matching; approximate string matching

\section{INTRODUCTION}

String matching is a long-standing field of discrete algorithms with numerous applications such as genome analysis [18], network surveillance [12] and cloud computing [14], where the need for privacy preservation is rapidly mounting.

Permission to make digital or hard copies of part or all of this work for personal or classroom use is granted without fee provided that copies are not made or distributed for profit or commercial advantage, and that copies bear this notice and the full citation on the first page. Copyrights for third-party components of this work must be honored. For all other uses, contact the owner/author(s). Copyright is held by the author/owner(s).

WPES'14, November 3, Scottsdale, Arizona USA

ACM 978-1-4503-3148-7/14/11.

http://dx.doi.org/10.1145/2665943.2665954.
In medical genomics, for example, oblivious string matching can be crucially relevant to clinical diagnoses based on sequenced data: recent work [2] has shown that the analysis of DNA or RNA reads, such as obtained through nextgeneration sequencing techniques, can provide a quick and relatively inexpensive way to test for the presence of specific pathogens in a patient's sample. In a number of cases, however, part or all the information needed to conduct the test, as well as the result itself, may need to be kept secret from the contributing parties. For commercial or safety reasons (e.g. bio-terrorism), the testing authority may need to keep the exact pathogen sequence private, while patients may not wish to trust a third-party conducting the test with their own genomic data or the status of their diagnosis. In such a scenario, the privacy must be kept rigorously on both sides, and cryptographic methods must be employed despite increased computational and communication costs. In cloud computing [14], an administrator may need to audit cloud access logs to detect malicious accesses. At the same time, it is not desirable to provide the administrator with access to users' private information. Oblivious string matching allows the administrator to audit the logs with complete privacy protection.

In string matching, an automaton holder has an automaton representing a regular expression while a text holder keeps a text string that needs to be tested. The text holder submits the text to the automaton holder letter by letter, triggering state transitions in the automaton, and finally obtains a notification of acceptance or rejection. Encryption allows us to perform the procedure obliviously, that is, with proof that the automaton holder has gained no knowledge about the text and the text holder no knowledge about the automaton and state transitions that occurred. Typically, several rounds of communication involving decryption and re-encryption are required to make a transition happen. An oblivious string matching method is evaluated by its computational cost for the automaton and text holders, communication cost and number of communication rounds.

A regular expression can be represented either by a DFA or an NFA. Troncoso et al. [24] and Frikken [5] designed oblivious string matching algorithms based on DFAs. An advantage of DFAs is that the active state is always unique and easy to keep track of. NFAs, on the other hand, have multiple active states leading to inferior run-time efficiency 
Table 1: Comparison of the complexity of oblivious NFA evaluation (ONE) to DFA counterparts. Here $n$ denotes the input text length, $|\Sigma|$ the size of alphabet, and $m$ the number of NFA states. The exponential factor $2^{m}$ is due to the fact that the number of DFA states can be exponentially larger than that of NFAs.

\begin{tabular}{c|ccc|ccc}
\hline Method & \multicolumn{3}{|c|}{ Preprocess } & \multicolumn{3}{c}{ Runtime } \\
& Computation & Communication & Round & Computation & Communication & Round \\
\hline Troncoso+ [24] & $O\left(2^{m}\right)$ & $O(|\Sigma|)$ & $O(1)$ & $O\left(n\left(2^{m}+|\Sigma|\right)\right)$ & $O\left(n\left(2^{m}+|\Sigma|\right)\right)$ & $O(n)$ \\
Frikken [5] & $O\left(n 2^{m}|\Sigma|\right)$ & $O\left(n 2^{m}|\Sigma|\right)$ & $O(1)$ & $O(n)$ & $O(1)$ & $O(1)$ \\
ONE & $O\left(m^{2}|\Sigma|+m^{3}\right)$ & $O\left(m^{2}|\Sigma|\right)$ & $O(1)$ & $O\left(n m^{2}\right)$ & $O\left(n m^{2}\right)$ & $O(n m)$ \\
\hline
\end{tabular}

in non-oblivious settings. The size of NFAs, however, can be exponentially smaller than DFAs. In this paper, we present an NFA-based oblivious string matching algorithm and prove it has better computational complexity bounds than DFA-based methods (Table 1). Our algorithm, termed ONE (Oblivious NFA evaluation), despite requiring a larger number of rounds, takes full advantage of NFA's compactness, making it exponentially faster than DFA-based algorithms in worst-case complexity. Furthermore, our algorithm allows us to trade some privacy for speed by selectively disclosing a part of the graph structure of NFA.

Approximate string matching (aka sequence alignment) is often implemented via dynamic programming (cf. NeedlemanWunsch and Smith-Waterman algorithms [18]). Such methods fill up a table with intermediate costs, where the computation of a cost involves integer comparison that cannot be implemented obliviously without Yao's garbled circuits [28]. We propose a novel oblivious technique based on Ukkonen NFAs [25]: a special grid-structured NFA. Existing algorithms either convert the NFA to a DFA [25] or require various manipulations beyond addition and multiplication [27]. ONE can be applied to Ukkonen NFA as well, but we will show that a protocol specially designed for Ukkonen NFA to improve computation and communication complexity by utilizing its regular grid structure. Both of our algorithms are secure against a semi-honest model.

The rest of this paper is organized as follows. Section 2 presents a matrix-based NFA evaluation in non-oblivious settings. Our privacy models and problem statements are defined in Section 3. The oblivious NFA evaluation protocol and its security proof are described in Section 4. Section 5 presents an oblivious protocol for Ukkonen NFA. Experimental results including applications to medical genomics are shown in Section 6. Section 7 concludes the paper.

\section{MATRIX-BASED NFA EVALUATION}

Additive homomorphic cryptosystems [21] allow us to perform addition of two integers without decryption. Oblivious multiplication protocols can be designed based on the cryptosystem, if several communication rounds are allowed [20]. When evaluating a NFA, active states are updated by following directed edges [25], typically implemented as pointers. However, following pointers in an oblivious manner is more difficult than performing addition and multiplication. Thus we propose the following NFA evaluation algorithm consisting solely of matrix calculations.

Let $\Sigma$ be a finite alphabet of letters and $\varepsilon$ be the empty string. A non-deterministic finite automaton $F=(V, E, \Theta, \Phi)$ is an edge-labeled directed graph, where $V=\{1, \ldots, m\}$ denotes a set of nodes called states, $E \subseteq V \times(\Sigma \cup\{\varepsilon\}) \times V$ is a set of labeled directed edges between states called transitions, and $\Theta$ and $\Phi$ represent the sets of start and accept states, respectively. If $F$ does not have $\varepsilon$-transitions, and all outgoing transitions are labeled with different letters, $F$ is a deterministic automaton. In a transition $(j, \sigma, k)$, we call $j$ the source state and $k$ the target state.

In this paper, we assume that the input NFA is a transitive closure NFA: if state $k$ is $\varepsilon$-reachable from state $j$, i.e., there exists a path connecting $j$ and $k$ consisting of $\varepsilon$-transitions only, then the automaton contains an $\varepsilon$-transition from $j$ to $k$. If the given NFA is not a transitive closure NFA, it can be computed in $O\left(\mathrm{~m}^{3}\right)$ time and $O\left(\mathrm{~m}^{2}\right)$ space [18].

Initially, only start states are active. As a text, $T=$ $T[1], \ldots, T[n]$, is given letter by letter, active states transition by following edges with the corresponding letter. Thus, when evaluating an NFA, one must track multiple active states simultaneously. All states connected to an active state with $\varepsilon$-transitions immediately turn active, and all start states always stay active. When at least one of the accept states is active at time $i$, the prefix $T[1 . . i]$ is accepted. Language $L(F)$ is the set of strings represented by all paths from start states to accept states. If $T[1 . . i]$ is accepted, a suffix of $T[1 . . i]$ belongs to $L(F)$.

Let us define a mask matrix $M[\sigma]$ as follows. If $(j, \sigma, k) \in$ $E, M[\sigma]_{j, k}=0$ and $M[\sigma]_{j, k}=1$ otherwise. It is the complement of the adjacency matrix restricted to letter $\sigma . M[\varepsilon]$ is a mask matrix for $\epsilon$-edges. The active state array is defined as $S \in \mathbb{Z}^{m}$, where $S[j]=0$ implies that the $j$-th state is active and otherwise inactive. Then, the state transition induced by letter $t$ is described as

$$
\hat{S}[k] \leftarrow \prod_{j=1}^{m}\left(S[j]+M[t]_{j, k}\right),
$$

for $k=1, \ldots, m$. It implies that $\hat{S}[k]=0$ iff there exists $j$ such that $S[j]=0$ and $M[t]_{j, k}=0$. Similarly, the $\epsilon-$ transition is realized using $M[\varepsilon]$.

Using the above update formulas, we describe the NFA evaluation method as follows. Initially, the active state array $S$ is set as

$$
S[j]= \begin{cases}0 & \text { if } j \in \Theta \\ 1 & \text { otherwise. }\end{cases}
$$

Before reading out the text, $\epsilon$-transition is performed once. At time $i$, the $i$-th letter $T[i]$ is given. In each iteration, the normal transition is applied and then $\epsilon$-transition is applied (eq. 1). All start states are reset to active, $\hat{S}[j]=0$ for $j \in \Theta$. If $\hat{S}[\phi]=0$ for some accept state $\phi \in \Phi$, the acceptance of the prefix is reported. This algorithm finishes in $O\left(m^{2} n\right)$ time and $O\left(\mathrm{~m}^{2}\right)$ space.

\section{OBLIVIOUS NFA EVALUATION}

\subsection{Privacy Model of NFAs}

Two stakeholders appear in our oblivious NFA evaluation problem: the text holder (TH) and automaton holder 
(AH). The text holder possesses a private text that cannot be shared with the automaton holder; the automaton holder possesses an NFA that must remain private too. The oblivious NFA evaluation (ONE) problem supposes that both parties cannot share their private information mutually, but they wish to jointly simulate the running of the NFA over the text. After evaluation, only the automaton holder (or the text holder) should learn the evaluation result, meanwhile, nothing but what can be inferred from the evaluation result should be learned from the protocol execution. Thus, the oblivious NFA evaluation problem can be defined as an instance of secure multiparty computation.

The notion of privacy for the text holder is obvious: any substring of the text should not be leaked by NFA evaluation. Privacy for the automaton holder, on the other hand, is subject to discussion. In the pioneering work of [24], where DFAs are used, the privacy of DFAs is defined as the initial state, the set of accept states, and the transition function. We extend this privacy definition of DFA to NFAs.

We can show that the privacy of an NFA, $F=(V, E, \Theta, \Phi)$, is only dependent on $E$, the labeled edges: no matter how the state indices, $V$, are set, the texts accepted by the NFA is unchanged. In other words, the language $L(F)$ is independent of $V$, and its disclosure does not leak any information on the NFA except its size, $|V|$. For the same reason, disclosure of the set of initial states $\Theta$ and accept states $\Phi$ do not leak any information about the NFA ${ }^{1}$.

Since the privacy of NFAs fully relies on the labeled edges, $E$, we define the privacy of $E$ by partitioning the mask matrix of labeled edges into a public and a private part:

$$
\begin{aligned}
M[\sigma]_{j, k}^{\text {pub }} & = \begin{cases}0 & \text { if } e=(j, \sigma, k) \in E \text { is public } \\
1 & \text { if } e=(j, \sigma, k) \notin E \text { is public } \\
\text { priv } & \text { otherwise. }\end{cases} \\
M[\sigma]_{j, k}^{\text {priv }} & = \begin{cases}0 & \text { if } e=(j, \sigma, k) \in E \text { is private } \\
1 & \text { if } e=(j, \sigma, k) \notin E \text { is private } \\
p u b & \text { otherwise. }\end{cases}
\end{aligned}
$$

where $j, k \in V$ and $\sigma \subseteq \Sigma \cup\{\epsilon\}$. In eq. 3 and eq. 4, "public" means that anyone, text holder included, can learn the presence or absence of edge $e=(j, \sigma, k)$, "private" means that only the automaton holder knows the presence or absence of $e$.

For example, $M[\sigma]_{j, k}^{\mathrm{pub}}=1$ indicates that the absence of edge $e=(j, \sigma, k)$ is publicly known, while $M[\sigma]_{j, k}^{\mathrm{pub}}=$ priv indicates that the text holder is not allowed to know the presence or absence of $e$ and $M[\sigma]_{j, k}^{\text {priv }}$ always takes 0 or 1 .

\subsection{Problem Statement}

With this matrix representation of privacy, the problem of oblivious NFA evaluation is immediately defined:

Definition 1. A text holder (TH) holds a private text of length $n, T \in \Sigma^{n}$. An automaton holder (AH) holds an NFA $F=(V, E, \Theta, \Phi)$ where $M[\sigma]^{\text {priv }}$ is the mask matrix representing the private partition of labeled edges in $F$. After execution of the protocol for oblivious NFA evaluation, AH

\footnotetext{
${ }^{1}$ More precisely, disclosure of $\Phi$ reveals the size of the accept state sets $|\Phi|$. However, by adding a special state that aggregates occurrence of acceptance, any NFA can be modified so that $|\Phi|=1$ always holds. So we can consider that nothing is revealed by disclosing $\Phi$.
}

learns the text size $n$ and whether $T[1 . . i]$ is accepted by $F$ for $i=1, \ldots, n$, but nothing else. TH learns the NFA size $|V|$ but nothing else.

We denote secure two-party computation $f$ by

$$
f\left(X_{1}, X_{2}\right) \longrightarrow\left(Y_{1}, Y_{2}\right)
$$

where $X_{1}$ and $X_{2}$ are private inputs from the first and second party, respectively; $Y_{1}$ and $Y_{2}$ are private outputs to the first and second party, respectively. Public inputs are omitted in this notation. With this notation, oblivious NFA evaluation can be written as:

$$
\operatorname{ONE}\left(T,\left\{M[\sigma]^{\text {priv }}\right\}_{\sigma \in \Sigma \cup\{\varepsilon\}}\right) \longrightarrow\left(|V|,\left(\left\{b_{i}\right\}_{i=1}^{n}, n\right)\right)
$$

where $b_{i} \in\{$ true, false $\}$ denotes whether text $T[1 . . i]$ is accepted by NFA $F$.

As shown in the definition, we are not hiding the sizes of the NFA, $|V|$, or the text, $n$. Because our protocols need to run on computers with specifically allocated physical memory space, it is difficult to conceal these sizes. By adding dummy states, obfuscation of such information is possible, it does however still give hints on $|V|$ or $n$, since the memory consumption cannot be made less than $O(|V|)$ or $O(n)$ for any obfuscation. If $\mathrm{AH}$ wishes to perfectly conceal all information about the input NFA, they can set all elements in $M[\sigma]^{\text {pub }}$ to private: $M[\sigma]_{j, k}^{\text {pub }}=$ priv for every $j, k \in V$.

We can easily modify the model to the model for the decision version of the problem. In this model, after execution of the protocol, AH learns only the fact that there are some substring of $T$ accepted by $F$ or not. This modification restricts reveal of information about $T$ to $\mathrm{AH}$.

The privacy model in this setting is almost equivalent to the one introduced in [24] with two differences: First, their approach basically defines privacy for DFAs only. NFAs are transformed into DFAs and the privacy is defined for the transformed DFAs. Second, our model allows the automaton holder to specify a private set of edges and edge labels of the NFA while the remaining public part can be published. In some NFAs with specific functionalities, such as Thompson NFAs or Ukkonen NFAs, the edges are known to form certain types of graphs. We will show that computation and communication complexity for oblivious evaluation of such NFAs can be greatly improved if the NFA's public part is appropriately specified.

\section{OBLIVIOUS NFA EVALUATION}

We introduce a secure computation that privately simulates NFAs, following the privacy model presented in the previous section. In this section, we assume that all elements of the input NFA are private, and introduce a naive protocol for oblivious protocol evaluation. Our oblivious NFA evaluation protocol is realized by simulating matrix-based NFA evaluation using the properties of homomorphic encryption.

\subsection{Cryptographic Primitives}

\subsubsection{Additively Homomorphic Encryption}

In a public key cryptosystem, encryption uses a public key that can be known to everyone, while decryption requires knowledge of the corresponding private key. Given such a pair (sk, pk) of private and public keys and a message $m$, then $c=\operatorname{Enc}_{\mathrm{pk}}(m ; \ell)$ denotes a (random) encryption, or ciphertext, of $m$, and $m=\operatorname{Dec}_{\text {sk }}(c)$ denotes decryption. If a public key cryptosystem guarantees that any 
two ciphertexts are indistinguishable, then this cryptosystem provides semantic security (IND-CPA security). An additive homomorphic cryptosystem allows addition operations on encrypted values without knowledge of the secret key. Specifically, there exists an operator "०" such that for any plaintexts $a_{1}$ and $a_{2}$ :

$$
\operatorname{Enc}_{\mathrm{pk}}\left(a_{1}+a_{2} ; \ell\right)=\operatorname{Enc}_{\mathrm{pk}}\left(a_{1} ; \ell_{1}\right) \circ \operatorname{Enc}_{\mathrm{pk}}\left(a_{2} ; \ell_{2}\right),
$$

where $\ell$ is uniformly random provided that at least one of $\ell_{1}$ and $\ell_{2}$ is. On the basis of this property, it follows that given a constant $a_{2}$ and the encryption $\operatorname{Enc}_{\mathrm{pk}}\left(a_{1} ; \ell\right)$, we can compute multiplications by $a_{2}$ via repeated application of "o". This also enables a re-randomization property, which allows the computation of a new random encryption $c^{\prime}=\operatorname{Enc}_{\mathrm{pk}}\left(a ; \ell^{\prime}\right)$ of $a$ from an existing encryption $c=\operatorname{Enc}_{\mathrm{pk}}(a ; \ell)$ of $a$, again without knowledge of the private key or of $a$, as follows:

$$
\operatorname{Enc}_{\mathrm{pk}}\left(a ; \ell^{\prime}\right)=\operatorname{Enc}_{\mathrm{pk}}\left(a ; \ell_{1}\right) \circ \operatorname{Enc}_{\mathrm{pk}}\left(0 ; \ell_{2}\right) \text {. }
$$

In the rest of the paper, we omit the random number $\ell$ from our encryptions for simplicity.

We require a cryptosystem that provides semantic security (under appropriate computational hardness assumptions), re-randomization, and the additive homomorphic property, such as the Paillier cryptosystem [21]. In addition, we make use of the following specific property of the Pailler encryption in our prptocol construction:

$$
\operatorname{Enc}_{\mathrm{pk}}\left(a_{1} ; \ell\right)^{-a_{2}}=\operatorname{Enc}_{\mathrm{pk}}\left(-a_{1} a_{2} ; \ell\right) .
$$

\subsubsection{Multiplication Protocol}

Paillier encryption only allows multiplication of two integers when either of the integers is not encrypted, as pointed out in section 4.1.1. Multiplication of two encrypted values by a party without the decryption key (referred to as sender) can be achieved if one round of communication with the party having the decryption key (referred to as evaluator) is allowed:

$$
\begin{array}{r}
\operatorname{MULT}\left(\left(\operatorname{Enc}_{\mathrm{pk}}\left(x_{1}\right), \operatorname{Enc}_{\mathrm{pk}}\left(x_{2}\right)\right), \mathrm{sk}\right) \rightarrow \\
\left(\operatorname{Enc}_{\mathrm{pk}}\left(x_{1} x_{2} \bmod N\right), \emptyset\right) .
\end{array}
$$

where sk is the decryption key corresponding to the Paillier encryption scheme used for the input. We call this problem MULT. A solution commonly used (e.g.: [20]) to problem MULT is as follows:

1. The sender computes Enc $\mathrm{pk}_{\mathrm{p}}\left(x^{\prime}\right)$ and $\operatorname{Enc}_{\mathrm{pk}}\left(y^{\prime}\right)$, and sends them to the evaluator:

$$
\begin{aligned}
& \operatorname{Enc}_{\mathrm{pk}}\left(x^{\prime}\right)=\operatorname{Enc}_{\mathrm{pk}}(x) \circ \operatorname{Enc}_{\mathrm{pk}}(u) \text { where } u \in_{r} \mathbb{Z}_{N} \\
& \operatorname{Enc}_{\mathrm{pk}}\left(y^{\prime}\right)=\operatorname{Enc}_{\mathrm{pk}}(y) \circ \operatorname{Enc}_{\mathrm{pk}}(v) \text { where } v \in_{r} \mathbb{Z}_{N}
\end{aligned}
$$

2. The evaluator decrypts $\operatorname{Enc}_{\mathrm{pk}}\left(x^{\prime}\right)$ and $\mathrm{Enc}_{\mathrm{pk}}\left(y^{\prime}\right)$ and sends $z^{\prime}=x^{\prime} y^{\prime} \bmod N$ to the sender.

3. The sender receives $z^{\prime}$, computes $\operatorname{Enc}_{\mathrm{pk}}(z)$ as follows, and outputs $\operatorname{Enc}_{\mathrm{pk}}(z)$.

$$
\begin{aligned}
\operatorname{Enc}_{\mathrm{pk}}(z)= & \operatorname{Enc}_{\mathrm{pk}}\left(z^{\prime}\right) \circ \operatorname{Enc}_{\mathrm{pk}}(y)^{-u} \\
& \circ \operatorname{Enc}_{\mathrm{pk}}(x)^{-v} \circ \operatorname{Enc}_{\mathrm{pk}}(u \cdot v)^{-1} \\
= & \operatorname{Enc}_{\mathrm{pk}}(x y \bmod N) .
\end{aligned}
$$

The evaluator can decipher all encrypted messages but they have all been obfuscated with random values; the sender only deals with encrypted messages that it cannot decipher. It is thus easy to see that neither the sender nor evaluator gain any information about $x_{1}$ and $x_{2}$ from the protocol execution.

\subsection{Oblivious NFA Evaluation Protocol}

We employ Paillier encryption as the encryption function in our protocol. When $A$ is an array, the $i$ th element is denoted by $A[i]$. $\operatorname{Enc}_{\mathrm{pk}}(A)$ denotes element-wise encryption of array $A$; the $i$ th element of $\operatorname{Enc}_{\mathrm{pk}}(A)$ denotes $\operatorname{Enc}_{\mathrm{pk}}(A[i])$. AH can arbitrarily specify the private part of NFA $F$ by setting $M[\sigma]^{\text {pri }}$ according to eq. 4 , while public information is set in $M[\sigma]^{\text {pub }}$ through eq. 3 .

Algorithm 1 gives a solution for problem ONE. First, the encrypted state variables are initialized (line 1-3): this step activates states contained in the set of initial states before NFA evaluation (eq. 11 in the algorithm corresponds to eq. $2)$. Then, initial $\epsilon$-transitions are performed (line 5-7).

Next, the text gets processed letter-by-letter (line 8-20). First, letter transitions are processed for all $(j, k)$ s.t. $M[T[i]]_{j, k}^{\mathrm{pub}} \neq$ 1 (line 11-13). Note that $M[T[i]]_{j, k}^{\mathrm{pub}}=1$ indicates that the absence of labeled edge $(i, T[i], j)$ is publicly known and, in such a case, the corresponding letter transition can be skipped. Then, $\epsilon$-transitions are processed in the same manner for all $(j, k)$ s.t. $M[T[i]]_{j, k}^{\text {pub }} \neq 1$ (line 15-17). The encrypted state array is updated by iterating over these two steps alternately for each letter.

In the following subsections, we describe both of these transition steps in greater details:

\subsubsection{Letter Transition}

Letter transitions are performed in the for-loop on line 11: TH updates state variables with eq. 15 and eq. 16 for edges $(j, T[i], k)$ s.t. $M[T[i]]_{j, k}^{\text {pub }} \neq 1$. This ensures that the letter transition is performed only for $T[i]$-labeled edges whose presence is known publicly or edges whose presence (or absence) is kept private.

In addition, due to the homomorphic property in eq. 15, the state $j$ is made active only when (1) state $k$ is active and (2) there exists a letter transition from state $k$ to state $j$. The result of this update is preserved in a temporary variable tmp. Note that this computation is oblivious to $\mathrm{TH}$. No matter if there exists a (private) edge between states $k$ and $j$, this update correctly evaluates the letter transition.

Next, using multiplication with protocol MULT, the state $j$ is made active if (1) tmp is active or (2) state $K$ is already active.

\subsection{2 $\varepsilon$-Transition}

$\epsilon$-transitions are performed in the for-loop at lines 5-15. Same as for the letter transitions, $\epsilon$-transitions are only performed for $\epsilon$-labeled edges whose absence is not known publicly. The mechanism of the update is exactly the same as with letter transitions.

\subsection{Correctness and Security of Oblivious NFA Evaluation}

In this subsection, we show that Algorithm 1 solves the oblivious NFA evaluation problem securely and correctly.

Theorem 1. Algorithm 1 correctly evaluates NFA F.

Intuitively, the theorem is proved by showing that active (zero) or inactive (non-zero) states, as updated by Algorithm 1 are always equivalent to the states computed by matrixbased NFA evaluation in Section 2. The values computed by Algorithm 1 are not exactly the same as the values computed 


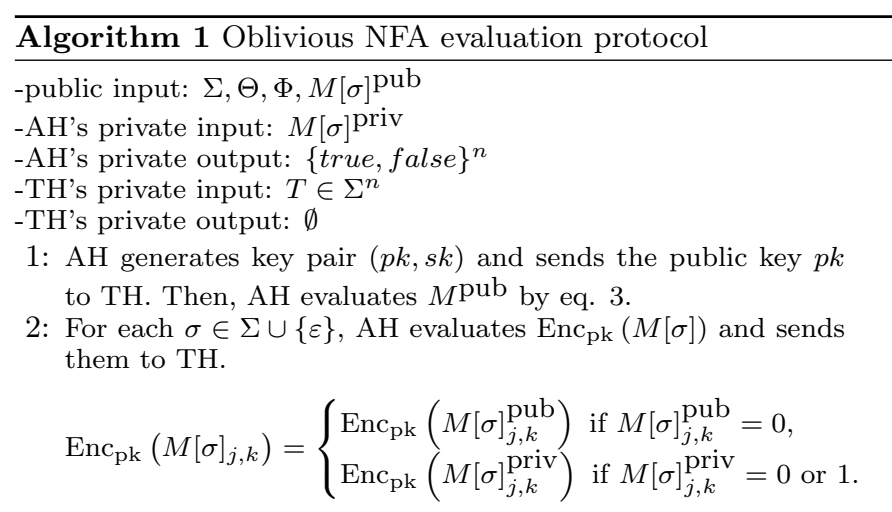

3: For each $1 \leq j \leq m, \mathrm{AH}$ initializes the array of the encrypted state variables $\operatorname{Enc}_{\mathrm{pk}}(S)$ and $\operatorname{Enc}_{\mathrm{pk}}(\hat{S})$ as follows and sends them to $\mathrm{TH}$.

$$
\begin{aligned}
\operatorname{Enc}_{\mathrm{pk}}(S[j]) & =\left\{\begin{aligned}
\operatorname{Enc}_{\mathrm{pk}}(0) & (j \in \Theta), \\
\operatorname{Enc}_{\mathrm{pk}}(1) & (\text { otherwise }) .
\end{aligned}\right. \\
\operatorname{Enc}_{\mathrm{pk}}(\hat{S}[j]) & =\operatorname{Enc}_{\mathrm{pk}}(1) .
\end{aligned}
$$

4: $\triangleright-\varepsilon$-transition-

5: for $(j, k) \in\left\{(j, k) \mid j, k \in V, M[\epsilon]_{j, k}^{\text {pub }} \neq 1\right\}$ do

6: $\quad \mathrm{TH}$ evaluates the following formula.

$t m p=\operatorname{Enc}_{\mathrm{pk}}(S[j]) \circ \operatorname{Enc}_{\mathrm{pk}}\left(M[\varepsilon]_{j, k}\right)$

$\operatorname{MULT}\left(\left(\operatorname{Enc}_{\mathrm{pk}}(\hat{S}[k]), t m p\right), \mathrm{sk}\right) \rightarrow\left(\operatorname{Enc}_{\mathrm{pk}}(\hat{\mathrm{S}}[\mathrm{k}]), \emptyset\right)$

7: end for

8: for $i=1$ to $n$ do

9: $\quad$ For each $1 \leq j \leq m$, TH initializes $\operatorname{Enc}_{\mathrm{pk}}(\hat{S})$ by $\hat{S}[j]=$ $\operatorname{Enc}_{\mathrm{pk}}(1)$.

10: $\triangleright-$-letter-transition-

11: $\quad$ for $(j, k) \in\left\{(j, k) \mid j, k \in V, M[T[i]]_{j, k}^{\text {pub }} \neq 1\right\}$ do

12: $\quad$ TH evaluates following formula.

$t m p=\operatorname{Enc}_{\mathrm{pk}}(S[j]) \circ \operatorname{Enc}_{\mathrm{pk}}\left(M[T[i]]_{j, k}\right)$

$\operatorname{MULT}\left(\left(\operatorname{Enc}_{\mathrm{pk}}(\hat{S}[k]), t m p\right), \mathrm{sk}\right) \rightarrow\left(\operatorname{Enc}_{\mathrm{pk}}(\hat{\mathrm{S}}[\mathrm{k}]), \emptyset\right)$

13: end for

14: $\triangleright-\varepsilon$-transition-

15: $\quad$ for $(j, k) \in\left\{(j, k) \mid j, k \in V, M[\epsilon]_{j, k}^{\text {pub }} \neq 1\right\}$ do

16: $\quad$ TH evaluates following formula.

tmp $=\operatorname{Enc}_{\mathrm{pk}}(S[j]) \circ \operatorname{Enc}_{\mathrm{pk}}\left(M[\varepsilon]_{j, k}\right)$

$\operatorname{MULT}\left(\left(\operatorname{Enc}_{\mathrm{pk}}(\hat{S}[k]), t m p\right), \mathrm{sk}\right) \rightarrow\left(\operatorname{Enc}_{\mathrm{pk}}(\hat{\mathrm{S}}[\mathrm{k}]), \emptyset\right)$

17: end for

18: $\quad$ For all $\phi \in \Phi$, TH sends $\operatorname{Enc}_{\mathrm{pk}}(\hat{S}[\phi])^{r_{0}}$ where $r_{0} \in_{r} \mathbb{Z}_{N}^{*}$ to $\mathrm{AH}$

19: $\quad$ AH decrypts $\operatorname{Enc}_{\mathrm{pk}}(\hat{S}[\phi])^{r_{0}}$ and outputs

$$
b_{i}= \begin{cases}\text { true } & \left(\text { if } r_{0} \hat{S}[\phi]=0\right) \\ \text { false } & \text { (otherwise) }\end{cases}
$$

20: end for by the matrix-based NFA evaluation. However, noting that acceptance is determined by the state (zero or non-zero), the proof simply shows that Algorithm 1 and the matrix-based NFA evaluation behave equivalently in the way states' active statuses are updated.

Theorem 2. If Paillier encryption is semantically secure and the text holder and automaton holder behave semihonestly, Algorithm 1 is secure in the sense of Definition 1.

We used the simulation-based standardized proof methodology in the semi-honest model. Intuitively, what $A H$ observes in the middle of the protocol execution is all additively or multiplicatively randomized; the TH's privacy is information-theoretic. What $T H$ observes in the middle of the protocol execution is all encrypted; since the encryption is semantically secure by assumption, AH's privacy is secure against probabilistic polynomial adversaries.

\subsection{Complexity Analysis}

This subsection discusses the complexity analysis of the ONE protocol in detail.

During initialization, Algorithm 1 encrypts the mask matrix $M[\sigma]$ in $O\left(m^{2}\right)$ time (line 2), and initializes encrypted state variables in $O(m)$ time (line 3 ).

We introduce the notation

$$
d=\left|\left\{(j, k) \mid \forall \sigma \in \Sigma, M[\sigma]_{j, k} \neq 1\right\}\right|,
$$

which denotes the number of transitions whose presence is public or whose presence (or absence) is kept private. Noting that TH can skip letter transitions and $\epsilon$-transitions when their absence is public, the protocol for ONE requires $O(d)$ transitions times per letter. A single transition invokes multiplications of encrypted values (addition in plaintext) and protocol MULT (multiplication in plaintext) $O(1)$ times. Letter- and $\epsilon$-transition cost $O(d)$ computation time and $O(d)$ communication rounds with communication of $O(d)$ words per letter. The protocol for ONE thus requires $O(n d)$ computation time, $O(n d)$ communication rounds, and communication of $O(n d)$ words to process a $n$-length text.

We note that transitions having different destination states can be processed independently. If we process transitions having different target states in one round, we can reduce communication rounds to $O(\ell)$ where $\ell$ is the maximum number of source states that a single state can have.

The upper bound of $d$ is $m^{2}$; this occurs in the perfect privacy setting (presence or absence of all transitions is kept private) defined by [24], yielding $O\left(n m^{2}\right)$ time, $O(n m)$ communication rounds, and $O\left(\mathrm{~nm}^{2}\right)$ word communication. If we can reveal the absence of transitions for a limited portion of all transitions, $d$ can be greatly reduced.

\subsection{Oblivious Regular Expression Matching}

We introduce an application of Algorithm 1 for the regular expression matching problem. The regular expression matching problem is given a regular expression $R$ and a text $T$, to report all occurrences of $R$ in $T$.

The Thompson NFA (TNFA for short) is a subclass of NFA proposed by Thompson [23]. Since TNFAs can be constructed from any regular expression using Thompson's construction algorithm, TNFAs are commonly used for general string matching. It is well known that every regular language is accepted by some TNFA [18]. In TNFAs, the letter 
transitions leaving state $j$ are always connected to state $j+1$ for every $j=1, \ldots, m$, whereas $\epsilon$-transitions can connect any two states. Given $R$, it is well known that the total number of states of the TNFA is linear in the size of $R$ [16].

Privacy Model of TNFA: Privacy model of TNFAs for oblivious evaluation is stated as follows. Let $F=(V, E, \Theta, \Phi)$ be the TNFA constructed from an input regular expression. Suppose the automaton holder holds a private TNFA and the text holder knows that the text is evaluated with a given TNFA (e.g., a given regular expression). Since letter transitions leaving state $j$ are always connected to state $j+1$ in a TNFA, it is obvious that letter transitions connecting state $j$ and state $k \neq j+1$ do not exist and the updates corresponding to these transitions can be skipped in our oblivious evaluation.

Formally, the privacy model of TNFAs is stated as follows. For every $\sigma \in \Sigma$ and every $j, k \in V$, the mask matrices $M^{\mathrm{pub}}[\sigma]$ and $M^{\mathrm{priv}}[\sigma]$ for the letter transitions of TFNAs are given by:

$$
\begin{aligned}
M^{\operatorname{pub}}[\sigma]_{j, k} & = \begin{cases}\text { priv } & \text { if } k=j+1 \\
1 & \text { otherwise. }\end{cases} \\
M^{\operatorname{priv}}[\sigma]_{j, k} & = \begin{cases}0 & \text { if }(j, \sigma, k) \in E \\
1 & \text { otherwise. }\end{cases}
\end{aligned}
$$

The mask matrix $M^{\mathrm{pub}}[\varepsilon]$ and $M^{\mathrm{priv}}[\varepsilon]$ for $\varepsilon$-transitions can be arbitrary determined depending on the privacy requirements of the automaton holder.

Protocol Description: Noting that letter transitions are only connecting state $j$ and state $j+1$, the letter transition part, lines 15-16 in Algorithm 1, can be modified as follows:

$$
\operatorname{Enc}_{\mathrm{pk}}(\hat{S}[j+1]) \leftarrow \operatorname{Enc}_{\mathrm{pk}}(S[j]) \circ \operatorname{Enc}_{\mathrm{pk}}\left(M[T[i]]_{j, j+1}\right)
$$

for $j=1, \ldots, m$. Since this update does not invoke the oblivious multiplication MULT, communication rounds required for letter transitions are reduced from $m^{2}$ to 0 .

If the automaton holder requires perfect privacy for $\epsilon$ transitions, the communication rounds still remains $O\left(\mathrm{~m}^{2}\right)$ to process private $\epsilon$ transitions. However, they can be reduced to $O(m)$ by assuming state $j$ is connected by $\epsilon$-transitions to at most a single state $k \neq j$. NFAs satisfying this condition belong to a subclass of TNFAs that does not necessarily support the full regular expression class, but contains useful subclasses of regular expression such as extended string patterns or prosite patterns [19].

Modification to the decision problem: The readers may be concerned that this algorithm reveals too much information to NFA holder. For this problem, we can modify the algorithm to the decision problem of regular expression matching by making small change to our algorithm. This problem is to report whether there are occurrences of regular expression $R$ in the input text $T$ or not. To solve this problem, we first add a self-loop transition labeled by all letters to the accepting state of the NFA, and then change the algorithm to output the encrypted accepting state $\hat{S}[\phi]$ only once at the end of computation. It is not hard to see that this modification yields that the NFA owner learns the result only at the end without the text owner's revealing any information about the input. This modification does not change the time and space complexity of our algorithm.

\section{OBLIVIOUS UKKONEN NFA EVALUA- TION PROTOCOL}

In this section, we introduce another useful class of string matching problem, called approximate string matching problem, which is extensively studied in text retrieval and bioinformatics $[18,19]$. For this problem, we propose a modification of the oblivious NFA evaluation protocol in section 4 to handle the oblivious approximate string matching problem. Using a class of NFAs with special structure, called Ukkonen NFAs [25, 27], this protocol drastically reduces the complexity of oblivious evaluation for approximate string matching.

\subsection{Approximate String Matching}

The edit distance between two strings $X$ and $Y$ is the minimum number of edit operations (a letter insertion, deletion, or substitution) required to convert $X$ into $Y$ or vice versa [18]. We denote the edit distance between $X$ and $Y$ as $e d(X, Y)$. The approximate string matching problem can be stated as follows:

Definition 2. The approximate string matching problem is, given a pattern $R \in \Sigma^{m}$, a text $T \in \Sigma^{n}$, and a threshold $k \geq 0$, to report all the end-position $j$ in $T$ of every substring $T[i . . j]$ that satisfies ed $(R, T[i . . j]) \leq k$.

A well-known approach to solve the approximate string matching problem is to use a dynamic programming algorithm, known as Smith-Waterman algorithm [22] or DPmatch algorithm [18]. The DP-based approach solves the problem of approximate string matching in $O(\mathrm{~nm})$ space and time. In general, secure comparison is expensive compared to secure arithmetic operations and unfortunately DP-based approach contains $O(\mathrm{~nm})$ comparisons. Thus, secure multiparty computation for approximate string matching based on the DP approach can be inefficient, particularly when the input text or pattern is large.

An alternative approach is to use the Ukkonen NFA (or UNFA) [25, 27]. The UNFA is an NFA that recognizes all substrings of an input text $T$ with edit distance at most $k$ against a pattern $R$. UNFA forms the regular $(k+1) \times(m+$ 1 )-grid structure, where $k$ is the maximum number of error allowed and $m$ is the length of a pattern $R$. In Figure 1, we show the Ukkonen NFAs for pattern $R=a b a b b$ with edit distance $k=2$.

Ukkonen [25] presented an algorithm that solves the approximate string matching problem in $O(n)$ time by converting Ukkonen NFA to DFA, and then by evaluating DFA on an input text. The drawback of this algorithm is space complexity caused by the exponential number $O\left(\min \left(3^{m}, m(2 m|\Sigma|)^{k}\right)\right)$ of DFA states [18]. Later, Wu an Manber [27] proposed an algorithm that directly simulates UNFA using bit-parallelism. We use Wu and Manber's NFA evaluation algorithm [27] as the basis of our protocol.

\subsection{Ukkonen NFA}

The Ukkonen NFA is a grid-structured NFA. Every row $\ell$ represents the number of errors, and every column $j$ represents the prefix of $P$. We denote by $U_{\ell, j}$ the state in row index $\ell$ and column index $j$, where $0 \leq \ell \leq k$ and $0 \leq j \leq m$. We call each edge labeled with $\Sigma$ a $\Sigma$-transition, which represents a letter transition with any letter of $\Sigma$.

As shown in Figure 1, a UNFA has the following classes of transitions: 1. Each horizontal letter transition (solid arrow with label $\sigma \in \Sigma$ ) from $U_{\ell, j}$ to $U_{\ell, j+1}$ represents matching of 


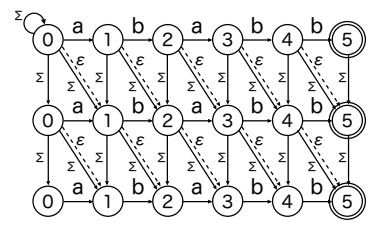

Figure 1: The Ukkonen NFA of $R=a b a b b, k=2$.

a letter $\sigma$ that is the $j$-th letter of $P$. We call this transition the $h$-trans. 2 . Each vertical $\Sigma$-transition (solid arrow with label $\Sigma$ ) from $U_{\ell, j}$ to $U_{\ell+1, j}$ inserts any letter to $P$ between the $(j-1)$-th and $j$-th letters. We call this transition the $v$-trans. 3. Each diagonal $\Sigma$-transition (solid arrow with label $\Sigma$ ) from $U_{\ell, j}$ to $U_{\ell+1, j+1}$ substitute a letter in $\Sigma$ for the $j$-th letter of $P$. We call this transition the $\Sigma$-d-trans. 4. Each diagonal $\varepsilon$-transition (dashed arrow with label $\varepsilon$ ) from $U_{\ell, j}$ to $U_{\ell+1, j+1}$ deletes the $j$-th letter of $P$. We call this transition the $\varepsilon$-d-trans. The rightmost states are accepting states. If the $j$ th accepting state from top reports acceptance, it indicates that the number of error is $j$.

\subsection{Oblivious Ukkonen NFA Evaluation}

In this subsection, we present an oblivious NFA evaluation protocol using the Ukkonen NFA.

Privacy Model of Oblivious UNFA: The privacy model of oblivious Ukkonen NFA evaluation is stated. Suppose the automaton holder holds a private UNFA and the text holder knows that the automaton holder evaluates the text with a certain UNFA (e.g., approximate string matching with a certain string). In this case, if the pattern length $m$ and maximum error threshold $k$ is public, the edge relations (not labels of edges) of the Ukkonen NFA is uniquely determined. The labels except h-transes are also uniquely determined. However, we note that the automaton holder's pattern to be matched need to be kept private.

Formally, the privacy model UNFAs is stated as follows. Let $F=(V, E, \Theta, \Phi)$ be UNFA constructed from input pattern $R$. From the structure of UNFA, for every $\sigma \in \Sigma \cup\{\varepsilon\}$ and $i, j \in V$, we set mask matrix $M^{\text {pub }}[\sigma]$ and $M^{\text {priv }}[\sigma]$ as follows:

$$
\begin{aligned}
& M^{\mathrm{pub}}[\sigma]_{j, k}= \\
& \begin{cases}\text { priv } & \text { if }(j, \sigma, k) \text { is h-trans, } \\
0 & \text { if }(j, \sigma, k) \text { is v-trans or } \Sigma \text { - or } \varepsilon \text {-d-trans, } \\
1 & \text { otherwise. }\end{cases} \\
& M^{\operatorname{priv}}[\sigma]_{j, k}= \begin{cases}0 & \text { if }(j, \sigma, k) \in E, \\
1 & \text { otherwise. }\end{cases}
\end{aligned}
$$

In oblivious evaluation of Ukknen NFAs, only the letters attached to h-trans from $U_{\ell, j}$ to $U_{\ell, j+1}$ are private and the remaining transitions and letters are all public.

Protocol Description: In Algorithm 2, we show the modified oblivious NFA evaluation protocol for Ukkonen NFA. The procedure basically follows Algorithm 1 except the update. The mask matrices are encrypted at line 2 . The encrypted state variables are initialized at line 3 .

States in the first row of the UNFA are updated only by htrans. This is processed by eq. 26. States not in the first row of the UNFA are updated by $v$-trans, $v$-trans, $\Sigma-d$-trans, and $\epsilon-d$-trans in turn at lines 8-12. In the algorithm, the results of update by h-trans (eq. 27) and v-trans (eq. 28) are integrated into $C 1$ by protocol MULT (eq. 31). Then,

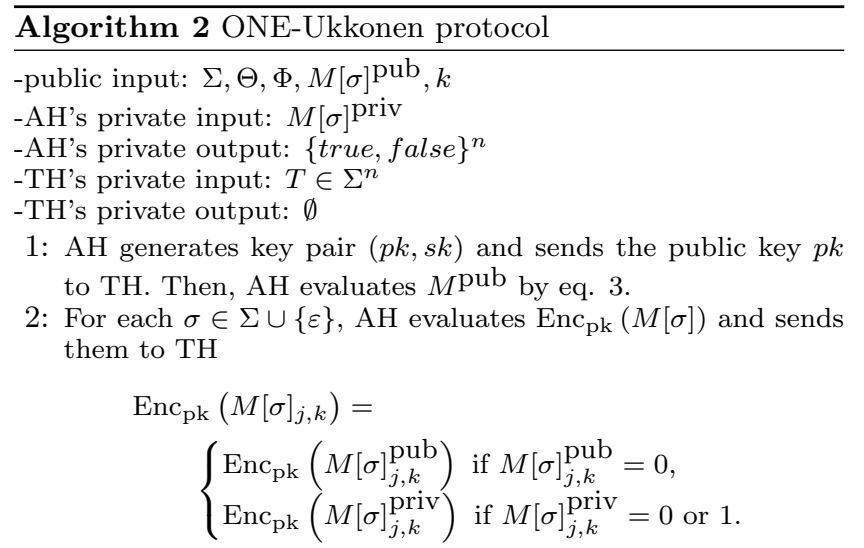

3: For each $0 \leq j \leq m$ and $0 \leq \ell \leq k, \mathrm{AH}$ initializes the array of the encrypted state variables $\operatorname{Enc}_{\mathrm{pk}}(S)$ and $\operatorname{Enc}_{\mathrm{pk}}(\hat{S})$ as follows and sends them to TH.

$$
\begin{aligned}
\operatorname{Enc}_{\mathrm{pk}}(S[\ell][j]) & =\left\{\begin{aligned}
\operatorname{Enc}_{\mathrm{pk}}(0) & \text { if } j=\ell=0 \\
\operatorname{Enc}_{\mathrm{pk}}(1) & \text { otherwise. }
\end{aligned}\right. \\
\operatorname{Enc}_{\mathrm{pk}}(\hat{S}[\ell][j]) & =\operatorname{Enc}_{\mathrm{pk}}(1) .
\end{aligned}
$$

4: for $i=1$ to $n$ do

5: $\quad$ TH evaluates $\operatorname{Enc}_{\mathrm{pk}}(S[0][0])=\operatorname{Enc}_{\mathrm{pk}}(0)$.

6: $\quad$ for $j=1$ to $m$ do

7: $\quad$ TH evaluates following formulas.

$$
\begin{aligned}
\operatorname{Enc}_{\mathrm{pk}}(\hat{S}[0][j]) \leftarrow & \operatorname{Enc}_{\mathrm{pk}}(S[0][j-1]) \\
& \circ \operatorname{Enc}_{\mathrm{pk}}\left(M[T[i]]_{U_{0, j}, U_{0, j+1}}\right)
\end{aligned}
$$

8: $\quad$ end for

9: $\quad$ for $\ell=1$ to $k$ do

10: $\quad$ for $j=1$ to $m$ do

11: $\quad$ TH evaluates following formula.

$$
\begin{aligned}
& B 1[j] \leftarrow \operatorname{Enc}_{\mathrm{pk}}(S[\ell][j-1]) \\
& \quad \circ \operatorname{Enc}_{\mathrm{pk}}\left(M[T[i]]_{U_{\ell, j}, U_{\ell, j+1}}\right) \\
& B 2[j] \leftarrow \operatorname{Enc}_{\mathrm{pk}}(S[\ell-1][j]) \\
& B 3[j] \leftarrow \operatorname{Enc}_{\mathrm{pk}}(S[\ell-1][j-1]) \\
& B 4[j] \leftarrow \operatorname{Enc}_{\mathrm{pk}}(\hat{S}[\ell][j-1]) \\
& \operatorname{MULT}((B 1[j], B 2[j]), \mathrm{sk}) \rightarrow(\mathrm{C} 1[\mathrm{j}], \emptyset) \\
& \operatorname{MULT}((B 3[j], B 4[j]), \mathrm{sk}) \rightarrow(\mathrm{C} 2[\mathrm{j}], \emptyset) \\
& \operatorname{MULT}((C 1[j], C 2[j]), \mathrm{sk}) \rightarrow\left(\operatorname{Enc}_{\mathrm{pk}}(\hat{\mathrm{S}}[\ell][\mathrm{j}]), \emptyset\right)
\end{aligned}
$$

12: end for

13: end for

14: $\quad$ For all $\phi \in \Phi$, TH sends $\operatorname{Enc}_{\mathrm{pk}}(\hat{S}[\phi])^{r_{0}}$ where $r_{0} \in_{r} \mathbb{Z}_{N}^{*}$ to $\mathrm{AH}$.

15: $\mathrm{AH}$ decrypts $\mathrm{Enc}_{\mathrm{pk}}(\hat{S}[\phi])^{r_{0}}$ and outputs

$$
b_{i}=\left\{\begin{array}{l}
\text { true if } r_{0} \hat{S}[\phi]=0, \\
\text { false otherwise }
\end{array}\right.
$$

16: end for 
the results of update by $\Sigma-d$-trans (eq. 29) and $\Sigma-\epsilon$-trans (eq. 30) are integrated into $C 2$ by protocol MULT (eq. 32). Finally, results $C 1$ and $C 2$ are integrated in eq. 33 . The calculations eq. 31 and eq. 32 can be performed in one round, and then perform eq. 33. By doing so, the number of rounds invoked by MULT is reduced from three to two.

Security. The security proof can be shown by similar arguments to that used in Sec. 4. The primitives are homomorphic addition and MULT protocol only. The TH's privacy is information theoretic and the AH's privacy is secure against probabilistic polynomial adversaries.

\subsubsection{Complexity Analysis}

The complexities of the proposed protocol are proved by the following theorem.

Theorem 3. For any input pattern $R$ and text $T$ on alphabet $\Sigma$, and threshold $k$, ONE-Ukkonen solves the oblivious approximate string matching in $O(k m n)$ time and $O\left(|\Sigma| m^{2}\right)$ preprocessing and space using $O(\mathrm{kmn})$ communication and $O(k n)$ rounds, where $m$ and $n$ are the pattern and text length.

Proof. First, we show the space complexity. The mask matrices $M[\sigma]^{\text {pub }}$ and $M[\sigma]^{\text {prib }}$ require $O\left(|\Sigma| m^{2}\right)$ space and the state array $S$ requires $O(\mathrm{~km})$ space. Thus, this algorithm consumes $O\left(|\Sigma| m^{2}\right)$ space and preprocessing in total. Next, we consider the update of each row of UNFA, which consists of $m$ states. From Algorithm 2, we observe that the update of a row for $\ell \geq 1$ requires one homomorphic additions and three MULT protocol invocation per state. The update for $\ell=0$ requires only one homomorphic addition per state. Furthermore, for each $\ell=0, \ldots k$ we see that only the $m$ MULT require communication, and they can be overlapped in one round. Combining the above discussion, we can update a row in $O(m)$ time, $O(m)$ communication, and one round. Since an UNFA consists of $k+1$ rows and a text contains $n$ letters, the result immediately follows.

\section{EXPERIMENTS}

This section includes benchmarking using simple regular expressions, and a medical genomic application to virus detection from short DNA reads.

\subsection{Benchmarking}

We benchmarked the communication and computational costs of ONE and ONE-Ukkonen in comparison to DFAbased approaches. For DFA-based approaches, we converted the NFAs into DFAs and used the DFAs for evaluation purposes. Frikken's method [5] is theoretically appealing but consumes huge memory space due to the exponential number of states necessary to implement Yao's garbled circuit [28]. We opted to compare ONE to the method proposed by Troncoso et al. [24] (hereafter noted as Troncoso).

Our Java implementations of ONE and Troncoso used Paillier cryptosystem with 1024 bit keys. The automaton holder and text holder resided in different PCs connected by $100 \mathrm{Mbit}$ ethernet. As shown in Section 3, the privacy of each transition can be defined separately. In the following, all transitions are assumed to be private so as to match Troncoso's privacy model. Table 2 describes the sizes of DFA and NFA that represents a regular expression $R=(a|b| c)^{*} a(a \mid b)^{t}$ for different values of $t^{2}$. The large difference in DFA and

\footnotetext{
${ }^{2}$ We use exponentiation to denote repetition, i.e. $a^{3}=a a a$.
}

Table 2: Sizes of DFA and NFA representing the regular expression $R=(a|b| c)^{*} a(a \mid b)^{t}$

\begin{tabular}{l|ccccc}
$\mathrm{t}$ & 0 & 1 & 5 & 10 & 15 \\
\hline NFA size & 2 & 3 & 7 & 12 & 17 \\
DFA size & 9 & 12 & 65 & 2063 & 68328
\end{tabular}

NFA sizes is reflected in computational and communication costs (Figure 2): Troncoso shows superexponential growth in the computational time for the automaton holder, while ONE's growth is subexponential, resulting in huge differences in total time.

Next, we tested the performance of ONE-Ukkonen presented in Section 5. A random text is matched against a regular expression $R=\left\{a b(a \mid b)^{*} b\right\}^{p}$ allowing at most two mismatches. Figure 3 shows communication and computational costs per letter $(p=1, \ldots, 4)$. Overall, ONE-Ukkonen performed exponentially better than Troncoso.

\subsection{Short Read Matching}

As mentioned previously, the field of medical genomics offer a number of real-life scenarios where some form of substring matching may need to be conducted in a privacypreserving framework.

Using short read data from [2], we simulated the oblivious detection of Norovirus genomic material in three samples from infected patients, using an additional non-infected sample for control. Each sample consists of a set of many millions short reads sequence (see table 3), each on average 150 letter-long, from which a small fraction can be mapped to a reference genome for human Norovirus GII (strain GII.4, Uniprot reference number D0EK97): that is, on average $1.175 \%$ and $0.013 \%$ (using approximate and exact matching, respectively) of all short read sequences are substrings of the whole Norovirus genome sequence (7646 letters, read in either direction).

Rather than testing for the match of each short read as a substring of the virus' genome, we selected a 31-letter substring from the genome, to use as our approximate pattern, against a randomly selected subset of the short reads. While the reads are generally not uniformly mapped on the reference genome, the "minimum coverage" observed with this RNA-Seq technique shows a lower-bound of approximately $0.02 \%$ of all reads matching any given position, allowing for up to two mismatches. By excluding known low-coverage areas (such as the extremities of the genome sequence), this lower-bound can be easily doubled. Therefore a pattern of 31 letters judiciously selected from the virus' sequence could be expected to match at least $0.024 \%$ of all reads. By selecting a random sample of 30,000 reads out of a maximum of $5,902,290$ for the largest sample, the worst-case probability of false negative on the test (failing to approximately match at least one read) is less than $0.074 \%$. Conversely, the length of the pattern, high specificity of viral RNA and background distribution of nucleotides ensure a negligibly low probability of false positive where none of the reads were a match for the viral pattern.

As shown in table 4, our implementation of ONE-Ukkonen described in section 5 , in $\mathrm{C}++$ using Paillier encryption with 512 bit keys, was able to perform on average each approximate substring match in $192.3 \mathrm{~s}$. (allowing for up to two mismatches), using a single Intel Xeon E5540 2.53GHz core. Using a 128-core cluster with parallelization, all 30,000 reads 

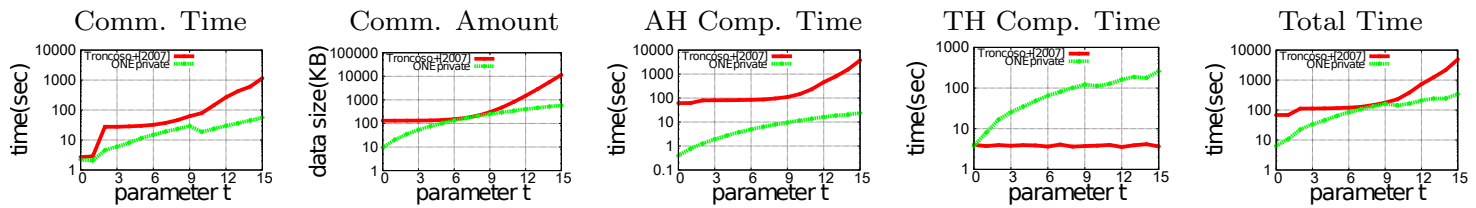

Figure 2: Computational and communication cost per text letter for regular expression $R=a\{a b\}(t)$. Green and red lines corresponds to ONE and Troncoso, respectively.
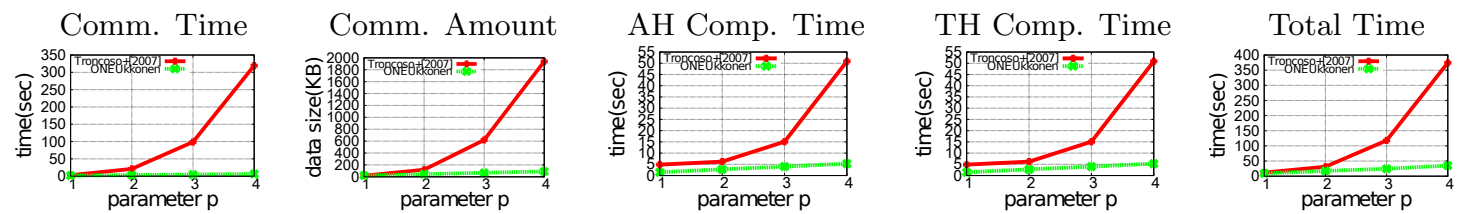

Figure 3: Computational and communication cost per text letter for approximate matching to regular expression $R=\left\{a b(a \mid b)^{*} b\right\}^{p}$. Green and red lines corresponds to ONE-Ukkonen and Troncoso, respectively.

Table 3: Total number of reads and size of the subset matching a 31-letter viral pattern, allowing for up to 0,1 and 2 mismatches respectively (figures in parentheses are averaged numbers of matches on a randomly subsampled set of 30,000 reads).

\begin{tabular}{|l|l|l|l|l|}
\hline Sample & $\begin{array}{l}\text { Total } \\
\text { reads }\end{array}$ & $\begin{array}{l}\text { Exact } \\
\text { Matches }\end{array}$ & $\mathrm{k}=1$ & $\mathrm{k}=2$ \\
\hline \multicolumn{5}{|c|}{ Norovirus samples } \\
\hline ERX202484 & 2957446 & $0(0)$ & $474(5.3)$ & $487(5.3)$ \\
ERX202485 & 5902290 & $1078(5.3)$ & $1122(5.3)$ & $1147(5.3)$ \\
ERX202486 & 2671055 & $0(0)$ & $0(0)$ & $1276(14)$ \\
\hline \multicolumn{5}{|c|}{ Control sample } \\
\hline ERX202487 & 3526904 & $0(0)$ & $0(0)$ & $0(0)$ \\
\hline
\end{tabular}

Table 4: Results of our implementation on randomly selected subsets of each sample, allowing for $k=1,2$ mismatches (all results are averaged over 3 samples).

\begin{tabular}{|l|c|c|}
\hline & $\mathrm{k}=1$ & $\mathrm{k}=2$ \\
\hline Sensitivity (true positives / total positives) & $2 / 3$ & $3 / 3$ \\
Specificity (true negatives / total negatives) & $1 / 1$ & $1 / 1$ \\
CPU time per read (1 core) & $129.2 \mathrm{~s}$ & $192.3 \mathrm{~s}$ \\
Time per sample (128 cores) & $8.4 \mathrm{~h}$ & $12.5 \mathrm{~h}$ \\
\hline
\end{tabular}

from the subsample were treated in less than 12.5 hours, each time correctly finding all positive matches present all samples. As our experimental results show, in all three infected samples, we were able to detect a significant number of viral RNA subsequences, identifying infected patients without disclosing any genomic data, nor the result of the test, to the other party.

\section{RELATED WORK AND DISCUSSION}

String matching with automaton evaluation covers a wide range of applications, such as exact matching, approximate matching, regular expression, etc [18]. In this paper, we present a protocol for oblivious NFA evaluation using matrixbased NFA evaluation.

Oblivious DFA evaluation has been extensively studied in various models over the recent years. [24] presented the first two-party oblivious DFA evaluation protocol based on oblivious transfer in the semi-honest model. In the same setting, [5] introduced an oblivious DFA evaluation based on Yao's garbled circuits with improved communication rounds and communication complexity. [3] considered techniques to outsource the computation of oblivious DFA evaluation without additional computational and communication costs. Oblivious DFA evaluation in the face of malicious adversaries was discussed in [15]. Extensions in the outsourced setting with homomorphic encryption was introduced by [26].

The semi-honest adversary model employed by our work follows [24] and [5]. Comparison of the computation, communication, and communication round complexities are summarized in Table 1. As already discussed, the computation and communication of existing protocols can be exponential when the target automaton is a NFA. Existing protocols are designed under the assumption that the target NFA can be transformed into a DFA, yet NFA-to-DFA transformation may require exponential computation time, and the resulting DFA may have an exponential number of states [1].

To the best of our knowledge, only ours and [13] provides oblivious NFA evaluation protocol. The NFA evaluation protocol presented in [13] is designed with an abstract computational environment referred to as the arithmetic black box model (ABB).The computation complexity of the protocol is $O\left(n m^{2}|\Sigma|\right)$, which is polynomial in the NFA size, whereas it dependent on the alphabet size. The communication and round complexity of this protocol cannot be specified without realization of the arithmetic black box model.

A number of studies have focused on polynomial-time secure multiparty computation specifically designed for specific string matching tasks; for example: exact string matching [11], string matching with wildcard and approximate string matching [8], sequence alignment by Smith-Waterman algorithm [10], hamming distance evaluation [9], and longest common sequence [7]. Because of the generality of the NFA, it covers a wide range of important string matching tasks, such as regular expression matching and approximate string matching. Our protocol successfully takes advantage of the generality of the NFA and provides a universal polynomialtime and space secure two-party solutions for these tasks.

Recent advances of secure computation techniques realizes secure computation in a practical time for some specific type of problems. While it is difficult to directly compare the computation or communication complexity of secure computation based on homomorphic encryption with secure circuit 
evaluation techniques, experimental comparison are needed, which is remained as our future work.

One of the problems remaining unsolved is the number of communication round, which is caused by multiple invocation of the one-round multiplication protocol. Classical homomorphic encryption only supports either additive or multiplicative homomorphism. Following Gentry's breakthrough [6], fully-homomorphic or somewhat homomorphic [17, 4] encryption schemes have been extensively discussed. Exploitation of such recent cryptographic techniques remains as an area to explore in our future work.

Acknowledgement The work is supported by JST CREST program "Advanced Core Technologies for Big Data Integration". Authors would like to thank the anonymous reviewers and Noboru Kunihiro for fruitful discussions.

\section{REFERENCES}

[1] A. V. Aho, J. E. Hopcroft, and J. D. Ullman. The Design and Analysis of Computer Algorithms. Addison-Wesley Publishing Company, 1974.

[2] E. M. Batty, T. N. Wong, A. Trebes, K. Argoud, M. Attar, D. Buck, C. L. Ip, T. Golubchik, M. Cule, R. Bowden, et al. A modified RNA-Seq approach for whole genome sequencing of RNA viruses from faecal and blood samples. PloS one, 8(6):e66129, 2013.

[3] M. Blanton and M. Aliasgari. Secure outsourcing of DNA searching via finite automata. Data and Applications Security and Privacy XXIV, pages 49-64, 2010.

[4] D. Boneh, C. Gentry, S. Halevi, F. Wang, and D. J. Wu. Private database queries using somewhat homomorphic encryption. In Applied Cryptography and Network Security, pages 102-118. Springer, 2013.

[5] K. B. Frikken. Practical private DNA string searching and matching through efficient oblivious automata evaluation. In Data and Applications Security XXIII, pages 81-94. Springer, 2009.

[6] C. Gentry. A fully homomorphic encryption scheme. PhD thesis, Stanford University, 2009.

[7] M. Gondree and P. Mohassel. Longest common subsequence as private search. In Proc. of $A C M$ workshop on Privacy in the electronic society, pages 81-90. ACM, 2009.

[8] C. Hazay and T. Toft. Computationally secure pattern matching in the presence of malicious adversaries. In Advances in Cryptology-ASIACRYPT 2010, pages 195-212. Springer, 2010.

[9] A. Jarrous and B. Pinkas. Secure hamming distance based computation and its applications. In Applied Cryptography and Network Security, pages 107-124. Springer, 2009.

[10] S. Jha, L. Kruger, and V. Shmatikov. Towards practical privacy for genomic computation. In IEEE Symposium on Security and Privacy 2008 (SP 2008), pages 216-230. IEEE, 2008.

[11] J. Katz and L. Malka. Secure text processing with applications to private dna matching. In $C C S^{\prime} 10$, pages 485-492. ACM, 2010.

[12] S. Kumar, S. Dharmapurikar, F. Yu, P. Crowley, and J. Turner. Algorithms to accelerate multiple regular expressions matching for deep packet inspection.
SIGCOMM Comput. Commun. Rev., 36(4):339-350, 2006.

[13] P. Laud and J. Willemson. Universally composable privacy preserving finite automata execution with low online and offline complexity. Cryptology ePrint Archive, Report 2013/678, 2013.

[14] J. Li, Q. Wang, C. Wang, N. Cao, K. Ren, and W. Lou. Fuzzy keyword search over encrypted data in cloud computing. In Proc. IEEE INFOCOM, pages 1-5, 2010.

[15] P. Mohassel, S. Niksefat, S. Sadeghian, and B. Sadeghiyan. An efficient protocol for oblivious dfa evaluation and applications. In Topics in Cryptology-CT-RSA 2012, pages 398-415. Springer, 2012.

[16] G. Myers. A four Russians algorithm for regular expression pattern matching. Journal of the ACM, 39(2):432-448, 1992.

[17] M. Naehrig, K. Lauter, and V. Vaikuntanathan. Can homomorphic encryption be practical? In Proc. of ACM workshop on Cloud computing security workshop, pages 113-124. ACM, 2011.

[18] G. Navarro and M. Raffinot. Flexible pattern matching in strings - practical on-line search algorithms for texts and biological sequences. Cambridge, 2002.

[19] G. Navarro and M. Raffinot. Fast and simple character classes and bounded gaps pattern matching, with applications to protein searching. Journal of Computational Biology, 10(6):903-923, 2003.

[20] K. Nissim and E. Weinreb. Communication efficient secure linear algebra. In TCC, pages 522-541, 2006.

[21] P. Paillier. Public-key cryptosystems based on composite degree residuosity classes. In Advances in cryptology EUROCRYPT, pages 223-238. Springer, 1999.

[22] T. F. Smith and M. S. Waterman. Identification of common molecular subsequences. Journal of molecular biology, 147(1):195-197, 1981.

[23] K. Thompson. Programming techniques: Regular expression search algorithm. CACM, 11(6):419-422, June 1968.

[24] J. R. Troncoso-Pastoriza, S. Katzenbeisser, and M. Celik. Privacy preserving error resilient DNA searching through oblivious automata. In $C C S^{\prime} 07$, pages 519-528. ACM, 2007.

[25] E. Ukkonen. Finding approximate patterns in strings. Journal of Algorithms, 6(1):132 - 137, 1985.

[26] L. Wei and M. K. Reiter. Third-party private dfa evaluation on encrypted files in the cloud. In Computer Security-ESORICS 2012, pages 523-540. Springer, 2012.

[27] S. Wu and U. Manber. Fast text searching: allowing errors. CACM, 35(10):83-91, Oct. 1992.

[28] A. C.-C. Yao. Protocols for secure computations. In FOCS'82, pages 160-164, 1982. 